\title{
ESTUDO DAS PROPRIEDADES FÍSICAS E QUÍMICAS DE MADEIRAS PROVENIENTES DE ESPÉCIES NATIVAS DA AMAZÔNIA LEGAL
}

Dayane Cristina Franzen ${ }^{1}$, Zaíra Morais dos Santos Hurtado de Mendoza ${ }^{2}$, Pedro Hurtado de Mendoza Borges ${ }^{3}$, Joao Paulo Sardo Madi ${ }^{4}$, Daiane de Souza Borges ${ }^{5}$

${ }^{1}$ Engenheira Florestal, Universidade Federal de Mato Grosso (UFMT). Cuiabá, Mato Grosso, Brasil.

${ }^{2} \operatorname{Prof}^{\mathrm{a}} \mathrm{Dr}^{\mathrm{a}}$ do Departamento de Engenharia Florestal. Universidade Federal de Mato Grosso (UFMT). Cuiabá, Mato Grosso, Brasil. (zairamorais09@gmail.com)

${ }^{3}$ Prof.Dr. do Departamento de Solos e Engenharia Rural. Universidade Federal de Mato Grosso (UFMT). Cuiabá, Mato Grosso, Brasil.

${ }^{4}$ Mestrando do Programa de Pós-graduação em Ciências Florestais e Ambientais, Universidade Federal de Mato Grosso (UFMT). Cuiabá, Mato Grosso, Brasil.

${ }^{5}$ Engenheira Florestal, Universidade Federal de Mato Grosso (UFMT). Cuiabá, Mato Grosso, Brasil.

\section{Recebido em: 06/04/2018 - Aprovado em: 10/06/2018 - Publicado em: 20/06/2018 DOI: 10.18677/EnciBio_2018A46}

\section{RESUMO}

A caracterização tecnológica da madeira consiste em determinar suas propriedades físicas, químicas, mecânicas e anatômicas, que são de grande importância, pois estão relacionados à transformação industrial, bem como à sua aptidão as mais variadas formas de utilização. O presente trabalho objetivou analisar algumas propriedades físico-químicas de quinze espécies nativas. Para as propriedades químicas quantificou-se os teores de extrativos solúveis em água fria e quente (norma ABCTP - M4/68), acetona (norma NBR - 14853), NaOH a 1\% (norma ABCTP - M5/68), e etanol/tolueno a uma mistura de 1:2 (ABTCP - M3/69) e também foi determinado o $\mathrm{pH}$ (NBR - 14339). Nas propriedades físicas foi analisado o teor de umidade (ABTCP - M2/71) e a massa básica específica das partículas (Foelkel et al., 1971) dessas espécies. Os maiores teores de extrativos solúveis em água fria, água quente, acetona, $\mathrm{NaOH} 1 \%$ e etanol/tolueno foram para a espécie de copaíba $(10,39 \%, 15,59 \%, 24,37 \%, 31,72 \%$ e $25,08 \%)$. Em relação ao $\mathrm{pH}$ o resultado variou numa escala de 3,5 (Cupiúba) a 5,63 (Tauari). Para as propriedades físicas das espécies estudadas, não ocorreu diferença significativa no teor de umidade, no entanto para a massa específica básica das partículas ocorreu diferença estatística. A análise de agrupamentos possibilitou classificar as espécies com base em suas características tecnológicas, mostrando sua similaridade ou semelhança entre elas. A caracterização físico-química dessas espécies servirá como uma ferramenta útil para aprimorar e/ou predizer usos distintos, além de abrir oportunidades para o reaproveitamento dessas espécies no setor florestal, auxiliando assim nas pesquisas cientificas e principalmente as empresas madeireiras.

PALAVRAS-CHAVE: Espécies florestais, extrativos, massa específica. 


\title{
STUDY OF THE PHYSICAL AND CHEMICAL PROPERTIES OF WOOD FROM NATIVE SPECIES OF THE LEGAL AMAZON
}

\begin{abstract}
The technological characterization of wood consists in determining its physical, chemical, mechanical and anatomical properties, which are of great importance, since they are related to the industrial transformation, as well as to its suitability in the most varied forms of use. The present work aimed to analyze some physicochemical properties of fifteen native species. For the chemical properties, the contents of extractives soluble in cold and hot water (ABCTP-M4 / 68 standard), acetone (standard NBR-14853), 1\% NaOH (ABCTP-M5 / 68 standard) and ethanol / toluene was added to a 1: 2 mixture (ABTCP-M3 / 69) and $\mathrm{pH}$ (NBR-14339) was also determined. In the physical properties the moisture content (ABTCP - M2 / 71) and the specific mass of the particles (Foelkel et al., 1971) of these species were analyzed. The highest contents of soluble extractives in cold water, hot water, acetone, $1 \% \mathrm{NaOH}$ and ethanol / toluene were for the copaiba species $(10.39 \%$, $15.59 \%, 24.37 \%, 31.72 \%$ and $25 \%, 08 \%)$. In relation to the $\mathrm{pH}$ the result varied in a scale of 3.5 (Cupiúba) to 5.63 (Tauari). For the physical properties of the species studied, there was no significant difference in moisture content, however for the specific basic mass of the particles a statistical difference occurred. The analysis of clusters made it possible to classify species based on their technological characteristics, showing their similarity or similarity between them. The physicochemical characterization of these species will serve as a useful tool to improve and / or predict different uses, as well as opening up opportunities for the reuse of these species in the forest sector, thus assisting scientific research and especially timber companies.
\end{abstract}

KEYWORDS: Forest species, extractive, specific mass.

\section{INTRODUÇÃO}

O Estado de Mato Grosso comercializou cerca de $\mathrm{R} \$ 5.411 .524 .257,24$ em espécies florestais no intervalo de 2004 a 2010, sendo que as espécies predominantes dessa comercialização eram oriundas do bioma amazônico, na qual $68,86 \%$ desse valor são representados pelas espécies de cambará, cupiúba, itaúba e angelim pedra (RIBEIRO et al., 2016).

Segundo dados do Centro das Indústrias Produtoras e Exportadoras de Madeira do Estado de Mato Grosso - CIPEM, o setor madeireiro é a quarta economia do estado com um PIB de 57.294.192, ou seja, 15\% do PIB industrial do Estado (CIPEM, 2012). Tendo em vista que o mercado quer permanecer nessa economia, o setor de base florestal tem buscado a diversificação na produção de produtos advindos da madeira, os principais produtos florestais comercializados pelo Estado de Mato Grosso é a madeira in natura (toras), madeira serrada e madeira beneficiada, segundo informações da Secretaria de Estado de Fazenda - SEFAZ, 2015.

No entanto, existe uma grande projeção de declino das florestas tropicais no mundo, gerada pelo intenso processo de desmatamento e exploração madeireira, apesar da possibilidade de ampliação da produção agrícola sem a necessidade de destruição das florestas remanescentes (MACEDO et al., 2012).

Para analisar as taxas de desmatamento na Amazônia legal, o governo brasileiro utiliza dados do projeto PRODES, que realiza o monitoramento via satélite 
por corte raso na Amazônia Legal, gerando taxas anuais estimadas pelo progresso de desmatamento constatado em cada imagem. Segundo dados do INPE, através do projeto PRODES, que é financiado pelo Ministério de Ciência, Tecnologia e Inovação - $\mathrm{MTCl}$ e conta com a colaboração do Instituto Brasileiro do Meio Ambiente e dos Recursos Naturais Renováveis (IBAMA) e do Ministério do Meio Ambiente (MMA), a taxa PRODES de desmatamento na Amazônia Legal em 2017 foi de $6624 \mathrm{~km}^{2}$, apresentando uma redução de 16\% em relação ao ano de 2016.

Fenômenos como esse, tem diminuído a oferta de madeira tropical no mercado, o que tem valorizado cada vez mais as madeiras de espécies nativas de crescimento lento, cujos preços tendem a permanecer em alta devido à intensa e crescente demanda consolidada em diversos setores econômicos (BRANCALION et al., 2012).

A demanda por espécies nativas advém do interesse e da busca por suas características tecnológicas, que nesse caso engloba as propriedades físicas, químicas, mecânicas e anatômicas. Esses fatores são preponderantes para determinadas finalidades na indústria florestal, e através do estudo dessas propriedades é possível adequar o uso das espécies de diferentes formas. Dados específicos sobre a composição química quantitativa das espécies tropicais brasileiras são escassos na literatura.

Cada espécie apresenta características químicas diversas, variando de acordo com a estrutura da madeira, esses compostos estão distribuídos de forma heterogênea e sujeito a estrutura anatômica da planta. Devido à diversidade de compostos químicos, pertencentes ao grupo dos extrativos, a quantificação dos mesmos, é feita com diferentes solventes, tanto polares como apolares. Em função disso, para a obtenção de madeira livre de extrativos, recomenda-se uma extração sucessiva para que a madeira possa ser utilizada para as análises de componentes da parede celular (MENDOZA, 2016).

Conforme Castro et al. (2013), a presença de elevados teores de extrativos é uma característica satisfatória para a produção de energia, devido ao poder calorífico desses componentes encontrado na madeira. Para Medeiros et al. (2014), a presença de extrativos na madeira é um ponto importante para a produção de carvão vegetal, pois eles aumentam sua densidade, favorecendo assim a produção de um carvão mais denso. Em relação às características físicas, a densidade básica de partículas e a umidade são propriedades da madeira que servem para indicar e diferenciar madeiras destinadas a diversos usos, como, construção civil, à fabricação de chapas ou à utilização na indústria de móveis.

A composição química quantitativa das espécies nativas brasileiras é rara na literatura, visto que essas pesquisas estão mais destinadas a florestas plantadas e não para florestas nativas. Logo o estudo das propriedades e caracterização químicas dessas espécies madeireiras é escasso, e por isso, tão importante para o setor de base florestal.

Portanto, o conhecimento da composição química e física da madeira é fundamental para o entendimento do comportamento deste material e para determinar seus usos como matéria-prima. Desta forma, o estudo aprofundado da madeira torna-se indispensável para sua utilização racional e efetiva nas necessidades da sociedade humana. Diante disso, o objetivo dessa pesquisa foi caracterizar química e fisicamente quinze espécies nativas da Amazônia Legal, em relação aos teores de extrativos solúveis em água (fria e quente), $\mathrm{NaOH}(1 \%)$, etanol/tolueno (1:2); acetona; $\mathrm{pH}$, massa específica básica das partículas e teor de 
umidade, visando o agrupamento dessas espécies de acordo com suas propriedades tecnológicas.

\section{MATERIAL E MÉTODOS}

Para a realização do estudo foram utilizadas 15 espécies nativas, provenientes do pátio de madeiras apreendidas do instituto de defesa agropecuária do estado do Mato Grosso (INDEA), localizado nas coordenadas geográficas $15^{\circ} 39^{\prime} 40.1$ "S 5558’26.9"W.

Dentro do pátio, as espécies estavam distribuídas em lotes, organizadas em forma de pilhas, e numeradas sequencialmente para posterior identificação. Essa identificação era realizada por peritos do próprio órgão, analisando-se a estrutura macroscópica das madeiras. Apesar de ter muitas madeiras no local, optou-se nesse estudo por trabalhar com as madeiras apreendidas no ano de 2014, 2015 e 2016. Cada espécie apreendida tinha um termo circunstanciado de ocorrência (TCO) único, referente a apreensões de lotes de madeiras, em momentos distintos por parte da autoridade policial.

Após a escolha aleatória dos lotes, foi separada e catalogada as quinze espécies nativas conforme especificado no quadro 1. Sequencialmente procedeu-se à coleta das amostras nos lotes de madeiras, amostrando-se uma tábua por espécie, resultando assim em 15 tábuas. A retirada das tábuas foi feita no interior da pilha, evitando-se possíveis problemas causados pelo intemperismo, também foram evitadas tábuas com defeitos, como rachaduras, trincas, tortuosidades, furos, nós, e atacadas por fungos e insetos.

QUADRO 1. Espécies e gêneros coletados para o estudo.

\begin{tabular}{|l|l|l|l|}
\hline \multicolumn{1}{|c|}{ Nome Vulgar } & \multicolumn{1}{c|}{ Ordem } & \multicolumn{1}{c|}{ Família } & \multicolumn{1}{c|}{ Gênero } \\
\hline Amescla Aroeira & Sapindales & Burseraceae & Protium sp. \\
\hline Angelin Amargoso & Fabales & Fabaceae & Vatairea sp. \\
\hline Cambará & Myrtales & Vochysiaceae & Qualea sp. \\
\hline Copaíba & Fabales & Fabaceae & Copaifera sp. \\
\hline Cumaru ferro & Fabales & Fabaceae & Dipteryx sp. \\
\hline Cumaru Rosa & Fabales & Fabaceae & Dipteryx sp. \\
\hline Cupiúba & Malpighiales & Goupiaceae & Goupia sp. \\
\hline Garrote & Rosales & Moraceae & Brosimum $s p$. \\
\hline Jatobá & Fabales & Fabaceae & Hymenaea $s p$. \\
\hline Jequetibá & Ericales & Lecythidaceae & Cariniana $s p$. \\
\hline Muiracatiara & Sapindales & Anacardiaceae & Astronium $s p$. \\
\hline Peroba Miúda & Gentianales & Apocynaceae & Aspidosperma $s p$. \\
\hline Roxinho & Fabales & Fabaceae & Peltogyne $s p$. \\
\hline Sucupira Preta & Fabales & Fabaceae & Bowdichia $s p$. \\
\hline
\end{tabular}




\begin{tabular}{|l|l|l|l|}
\hline Tauari & Ericales & Lecythidaceae & Couratari sp. \\
\hline
\end{tabular}

Posteriormente cada tábua foi seccionada em cinco peças de $30 \mathrm{~cm}$ e redimensionada para as dimensões de $8 \mathrm{~cm} \times 2 \mathrm{~cm} \times 25 \mathrm{~cm}$, correspondendo respectivamente a largura, espessura e comprimento, totalizando ao final 75 amostras para os ensaios de composição química e composição física. Em seguida as peças menores foram transformadas manualmente em palitos, que foram moídas e deram origem à serragem, que foi classificada nas peneiras de 40 e 60 mesh para os ensaios, utilizando-se o material que ficou retido na peneira de 60 mesh.

Os ensaios químicos e físicos foram realizados no laboratório de Tecnologia Química de Produtos Florestais, pertencente à Faculdade de Engenharia Florestal (FENF), da Universidade Federal de Mato Grosso (UFMT) - Campus Cuiabá, e seguiram as metodologias descritas no quadro 2 .

QUADRO 2. Normas utilizadas nas análises químicas e físicas.

\begin{tabular}{|l|l|}
\hline \multicolumn{1}{|c|}{ Análises } & \multicolumn{1}{c|}{ Norma } \\
\hline Amostragem e Processamento do Material & ABTCP - M1/71 \\
\hline Determinação do Teor de Extrativos em Água Fria & ABTCP - M4/68 \\
\hline Determinação do Teor de Extrativos em Água Quente & ABTCP - M4/68 \\
\hline Determinação do Teor de Extrativos em NaOH (1\%) & ABTCP - M5/68 \\
\hline Determinação do Teor de Extrativos em Acetona & NBR - 14853 \\
\hline Determinação do Teor de Extrativos em etanol/Tolueno & ABTCP - M3/69 \\
\hline Determinação do pH & NBR - 14339 \\
\hline Massa específica básica das Partículas & Foelkel et al., (1971) \\
\hline Determinação do teor de umidade & ABTCP - M2/71 \\
\hline
\end{tabular}

Para os ensaios de água fria, água quente, $\mathrm{NaOH}(1 \%)$, acetona, teor de umidade, $\mathrm{pH}$ e massa específica básica da partícula foram realizadas três repetições e para a variável etanol/tolueno (1:2) foram duas repetições, devido a falta do reagente tolueno em laboratório.

$O$ processamento analítico dos dados $\mathrm{e}$ as tabelas foram elaborados utilizando-se a planilha eletrônica EXCEL (2016). A análise de variância (ANOVA) e a comparação de médias foram realizadas com o programa $R$, versão 1.0.44, adotando-se os testes $F$ (Fisher) e Scott Knott $(p \leq 0,05)$, respectivamente. Com o referido programa aplicou-se também a técnica multivariada de agrupamento ("Cluster") para verificar a semelhança entre as espécies.

Antes de aplicar a técnica de agrupamentos, os dados foram padronizados com o propósito de eliminar a influência de unidades diferentes. Em seguida foi elaborado o dendrograma, cujo diagrama de árvore foi exibido de acordo com os grupos formados considerando-se as propriedades químicas e físicas para as quinze espécies estudadas. Na construção final dos dendrogramas, o nível de similaridade foi medido ao longo do eixo vertical e as diferentes observações foram listadas ao longo do eixo horizontal.

\section{RESULTADOS E DISCUSSÃO}



$1)$.

Após a análise dos dados obteve-se o resumo da análise de variância (Tabela

TABELA 1. Resumo da análise de variância para as características físico-químicas estudadas.

\begin{tabular}{lccr}
\hline \multicolumn{1}{c}{ Análises Químicas e Físicas } & QM & $\begin{array}{c}\text { Probabilidade } \\
(\text { Pr>FC) }\end{array}$ & $\begin{array}{c}\text { CV } \\
(\%)\end{array}$ \\
\hline Água Fria & 19,753 & $5,98 \mathrm{E}-07$ & 22,64 \\
Água Quente & 34,438 & $3,75 \mathrm{E}-08$ & 15,65 \\
NaOH (1\%) & 124,87 & $1,92 \mathrm{E}-13$ & 8,62 \\
Etanol Tolueno (1:2) & 51,350 & $1,18 \mathrm{E}-08$ & 6,06 \\
pH & 0,9013 & $2,99 \mathrm{E}-12$ & 3,23 \\
Massa Específica Básica das Partículas & 0,0038 & $2,05 \mathrm{E}-19$ & 2,53 \\
Teor de Umidade & 1,720 & 0,0022 & 11,18 \\
\hline
\end{tabular}

QM: Quadrado médio; CV (\%): Coeficiente de variação.

Observa-se na Tabela 1 que todos os valores da probabilidade foram menores que 0,05 , logo rejeita-se a hipótese nula e conclui-se que existe diferença entre as médias das espécies estudadas de cada tratamento. No entanto, o teor de umidade na análise de variância foi significativo, havendo assim diferença entre as médias das espécies nesse tratamento, porém ao realizar o teste de Scott Knott para comparações de médias, as mesmas foram classificadas como não havendo diferença entre si, isso ocorreu, provavelmente, pela exigência deste teste.

Após a análise de variância efetuou-se a análise descritiva e o teste de médias, para os teores de extrativos (Tabela 2) e para o $\mathrm{pH}$, massa específica básica das partículas e teor de umidade (Tabela 3). Conforme a Tabela 2, ao aplicar o teste de comparação entre as médias de Skott knott observou-se que ocorreu diferença estatística entre as espécies, sendo que a copaíba se destacou em todos os tipos de extração (10,39\%; $15,59 \% ; 31,72 \% ; 25,09 \%$ e $24,37 \%)$ respectivamente. Por outro lado, a espécie que apresentou um menor teor de extrativos foi o tauari $(1,25 \% ; 1,43 \% ; 5,17 \% ; 4,1 \% ; 2,14 \%)$ nesta ordem.

Braz et al., (2014), trabalhando com madeira do cruzamento de Eucaliptus urophylla com Eucaliptus grandis de 30 a 35 meses, encontraram valores de extrativos solúveis em álcool/tolueno de 0,70 a $1,07 \%$, sendo bem abaixo dos dados dessa pesquisa. Chagas et al. (2014), trabalhando com madeira de teca de 4, 6 e 12 anos, encontraram valores de extrativos solúveis em álcool/tolueno variando de 3,59 a $4,76 \%$. Esses valores são próximos aos encontrados nessa pesquisa para a espécie Tauari (4,10\%). Ferreira et al (2015), analisando o teor de extrativos em madeira de teca aos 13 anos de idade, encontraram valores de 3,44\% e de $9,66 \%$ para extrativos solúveis em água fria e quente respectivamente. Esses valores foram próximos aos encontrados nesse estudo para a madeira de jequitibá $(4,11 \%$ - água fria) e cupiúba (9,95\% - água quente). 
TABELA 2. Resultados da análise descritiva e do teste de médias para os teores de extrativos conforme o tipo de solvente.

\begin{tabular}{|c|c|c|c|c|c|}
\hline \multirow{2}{*}{ Nome Vulgar } & \multicolumn{5}{|c|}{ Teor de Extrativos } \\
\hline & Água Fria & Água Quente & $\mathrm{Na} \mathrm{OH}_{(1 \%)}$ & Etanol/Tolueno & Acetona \\
\hline Copaíba & $10,39^{ \pm 0,82}(7,9) \mathrm{a}$ & $15,59^{ \pm 0,54}(3,45) a$ & $31,72^{ \pm 1,08}(3,39) a$ & $25,09^{ \pm 0,51}(2,02) a$ & $24,37^{ \pm 0,62}(2,55) \mathrm{a}$ \\
\hline Angelin- Amargoso & $5,15^{ \pm 1,92}(37,3) b$ & $7,1^{ \pm 2,4}(33,8) d$ & $16,34^{ \pm 1,94}(11,8) d$ & $16,34^{ \pm 0,75}(4,61) d$ & $7,64^{ \pm 0,62}(8,05) c$ \\
\hline Cumaru-Ferro & $5,82^{ \pm 1,06}(18,2) b$ & $10,05^{ \pm 1,06}(10,5) c$ & $19,93^{ \pm 1,10}(5,53) c$ & $16,05^{ \pm 0}(0) b$ & $10,05^{ \pm 0,53}(5,26) b$ \\
\hline Cupiúba & $7,28^{ \pm 0,53}(7,32) b$ & $9,95^{ \pm 1,92}(19,3) c$ & $16,87^{ \pm 1,41}(8,36) \mathrm{e}$ & $10,12^{ \pm 0,50}(4,96) d$ & $6,57^{ \pm 0,31}(4,68) c$ \\
\hline Garrote & $6,44^{ \pm 0,31}(4,81) b$ & $12,54^{ \pm 1,12}(8,91) c$ & $18,6^{ \pm 0,82}(4,41) d$ & $11,09^{ \pm 0,25}(2,28) d$ & $2,15^{ \pm 1,55}(72,1) \mathrm{e}$ \\
\hline Jatobá & $6,93^{ \pm 1,23}(17,8) b$ & $12,08^{ \pm 1,07}(8,82) b$ & $24,16^{ \pm 0,81}(3,37) b$ & $14,21^{ \pm 0,75}(5,3) c$ & $7,28^{ \pm 1,07}(14,6) c$ \\
\hline Roxinho & $5,45^{ \pm 1,85}(34) b$ & $7,56^{ \pm 0,3}(4,03) d$ & $18,45^{ \pm 1,22}(6,6) d$ & $10,72^{ \pm 0,50}(4,64) d$ & $6,33^{ \pm 1,61}(25,4) c$ \\
\hline Amescla-Aroeira & $4,43^{ \pm 1,23}(27,7) \mathrm{C}$ & $8,33^{ \pm 0,81}(9,75) c$ & $24,29^{ \pm 1,87}(7,69) b$ & $10,99^{ \pm 1}(9,13) d$ & $10,28^{ \pm 1,63}(15,8) b$ \\
\hline Jequitibá & $4,11^{ \pm 0,31}(7,54) \mathrm{c}$ & $6,08^{ \pm 0,93}(15,3) d$ & $14,49^{ \pm 0,62}(4,28) \mathrm{e}$ & $11,99^{ \pm 0,31}(4,22) d$ & $8,77^{ \pm 1,07}(12,2) b$ \\
\hline Cumaru- Rosa & $2,49^{ \pm 0,31}(12,4) d$ & $6,41^{ \pm 1,63}(25,4) d$ & $15,66^{ \pm 4,03}(25,7) \mathrm{e}$ & $8,54^{ \pm 1,01}(11,8) \mathrm{e}$ & $3,02^{ \pm 1,87}(62,1) \mathrm{e}$ \\
\hline Muiracatiara & $2,86^{ \pm 0,54}(18,8) d$ & $8,77^{ \pm 0,93}(10,6) c$ & $21,82^{ \pm 0,62}(2,84) c$ & $11,63^{ \pm 0,51}(4,35) d$ & $9,66^{ \pm 1,88}(19,5) b$ \\
\hline Peroba-Miúda & $2,72^{ \pm 0}(0) d$ & $4,35^{ \pm 0,54}(12,5) d$ & $12,14^{ \pm 1,13}(9,32) f$ & $6,34^{ \pm 0,26}(4,04) f$ & $4,53^{ \pm 0,83}(18,3) d$ \\
\hline Sucupira- Preta & $2,67^{ \pm 1,72}(64,3) d$ & $5,34^{ \pm 1,34}(25,2) d$ & $11,39^{ \pm 0,53}(4,69) f$ & $6,23^{ \pm 1,26}(20,2) f$ & $3,91^{ \pm 0,53}(13,7) d$ \\
\hline Cambará & $0,88^{ \pm 0,61}(69,1) \mathrm{e}$ & $5,79^{ \pm 1,05}(18,2) d$ & $11,93^{ \pm 1,10}(9,18) f$ & $6,14^{ \pm 0,74}(12,1) f$ & $4,56^{ \pm 1,10}(24) d$ \\
\hline Tauari & $1,25^{ \pm 0,31}(24,7) \mathrm{e}$ & $1,43^{ \pm 1,11}(77,8) \mathrm{e}$ & $5,17^{ \pm 0,31}(5,97) \mathrm{g}$ & $4,1^{ \pm 0}(0) \mathrm{g}$ & $2,14^{ \pm 1,41}(66,1) \mathrm{e}$ \\
\hline
\end{tabular}

Obs: - $\mathrm{NaOH}(1 \%)$ : Teor de extrativos em Hidróxido de sódio (1\%); Expoentes precedidos de \pm representam o desvio padrão e valores entre parêntesis correspondem ao coeficiente de variação; Médias seguidas de letras iguais nas colunas não diferem entre si pelo teste de Scott Knott $(p \unlhd), 05)$. 
TABELA 3. Resultados da análise descritiva e o teste de médias para o $\mathrm{pH}$, a massa especifica básica de partículas e o teor de umidade.

\begin{tabular}{llll}
\hline Nome Vulgar & pH & Massa básica & Teor de Umidade \\
\hline Tauari & $5,63^{ \pm 0,21}(3,7) \mathrm{a}$ & $0,15^{ \pm 0,005}(3,25) \mathrm{f}$ & $6,5^{ \pm 1,0}(15,4) \mathrm{a}$ \\
Amescla- Aroeira & $4,77^{ \pm 0,06}(1,21) \mathrm{b}$ & $0,23^{ \pm 0,006}(2,7) \mathrm{c}$ & $6,0^{ \pm 0,5}(8,33) \mathrm{a}$ \\
Copaíba & $4,57^{ \pm 0,21}(4,56) \mathrm{b}$ & $0,19^{ \pm 0,015}(7,83) \mathrm{e}$ & $7,0^{ \pm 1,0}(14,3) \mathrm{a}$ \\
Cumaru- Ferro & $4,67^{ \pm 0,06}(1,27) \mathrm{b}$ & $0,26^{ \pm 0,003}(1,27) \mathrm{a}$ & $5,5^{ \pm 0,5}(9,09) \mathrm{a}$ \\
Cumaru- Rosa & $4,57^{ \pm 0,21}(4,56) \mathrm{b}$ & $0,21^{ \pm 0,004}(1,85) \mathrm{d}$ & $6,33^{ \pm 0,76}(12,1) \mathrm{a}$ \\
Garrote & $4,53^{ \pm 0,15}(3,37) \mathrm{b}$ & $0,19^{ \pm 0,004}(1,86) \mathrm{e}$ & $6,83^{ \pm 0,29}(4,22) \mathrm{a}$ \\
Jatobá & $4,63^{ \pm 0,12}(2,49) \mathrm{b}$ & $0,22^{ \pm 0,001}(0,48) \mathrm{c}$ & $6,16^{ \pm 0,29}(4,68) \mathrm{a}$ \\
Muiracatiara & $4,4^{ \pm 0}(0) \mathrm{b}$ & $0,26^{ \pm 0,005}(2,09) \mathrm{a}$ & $6,83^{ \pm 0,76}(11,2) \mathrm{a}$ \\
Peroba- Miúda & $4,83^{ \pm 0,35}(7,27) \mathrm{b}$ & $0,15^{ \pm 0,005}(3,28) \mathrm{f}$ & $8,0^{ \pm 0,87}(10,8) \mathrm{a}$ \\
Angelin- Amargoso & $4,1^{ \pm 0}(0) \mathrm{c}$ & $0,24^{ \pm 0,006}(2,53) \mathrm{b}$ & $6,16^{ \pm 0,76}(12,4) \mathrm{a}$ \\
Cambará & $4,07^{ \pm 0,05}(1,26) \mathrm{c}$ & $0,22^{ \pm 0,003}(1,53) \mathrm{c}$ & $5,0^{ \pm 0,87}(17,3) \mathrm{a}$ \\
Roxinho & $4^{ \pm 0}(0) \mathrm{c}$ & $0,26^{ \pm 0,001}(0,46) \mathrm{a}$ & $5,16^{ \pm 1,04}(20,1) \mathrm{a}$ \\
Sucupira- Preta & $3,8^{ \pm 0}(0) \mathrm{d}$ & $0,26^{ \pm 0,004}(1,59) \mathrm{a}$ & $6,33^{ \pm 0,29}(4,56) \mathrm{a}$ \\
Cupiúba & $3,5^{ \pm 0}(0) \mathrm{e}$ & $0,25^{ \pm 0,002}(0,88) \mathrm{a}$ & $6,16^{ \pm 0,29}(4,68) \mathrm{a}$ \\
Jequitibá & $3,6^{ \pm 0}(0) \mathrm{e}$ & $0,24^{ \pm 0,005}(2,14) \mathrm{b}$ & $6,83^{ \pm 0,58}(8,45) \mathrm{a}$ \\
\hline
\end{tabular}

Obs: Expoentes precedidos de \pm representam o desvio padrão e valores entre parêntesis correspondem ao coeficiente de variação; Médias seguidas de letras iguais nas colunas não diferem entre si pelo teste de Scott Knott (p $₫$ ),05).

Diante do exposto na Tabela 3 considerando-se o pH como meio de análise, observou-se que houve diferença estatística entre as espécies. Neste ensaio constatou-se que a madeira com o maior valor de $\mathrm{pH}$ foi o tauari $(5,63)$, por outro lado, a cupiúba foi a que obteve o menor valor de $\mathrm{pH}(3,50)$, confirmando estudos de Stamm (1964) acerca do $\mathrm{pH}$ que variaram de 3,0 a 5,5 para madeiras.

Quanto à massa específica básica das partículas, houve diferença estatística entre as espécies. A muiracatiara, roxinho, cumaru ferro e sucupira preta foram as que apresentaram o maior valor $\left(0,26 \mathrm{~g} / \mathrm{cm}^{3}\right)$, enquanto que 0 menor valor foi constatado nas espécies de peroba miúda e tauari $\left(0,15 \mathrm{~g} / \mathrm{cm}^{3}\right)$. Ao analisar o teor de umidade, não apresentou diferença estatística entre as espécies, esse fato pode ser explicado devido estas já terem secado naturalmente ao ar livre. Nessas condições, todas atingiram o seu ponto de equilíbrio de umidade com o ar. Ao realizar a análise de agrupamentos através dos dados obtidos em laboratório, elaboraram-se quatro dendrogramas para cada variável estudada (Figura 1 e 2). 


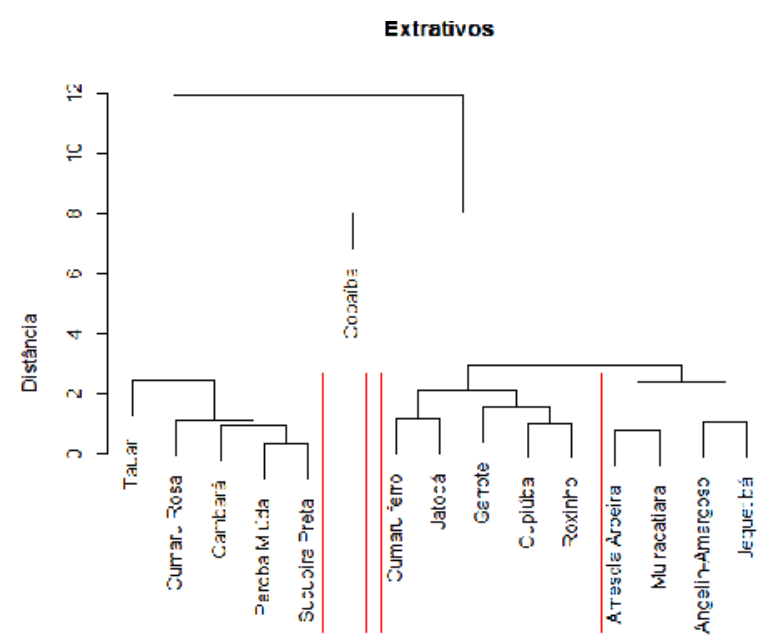

Fspéries

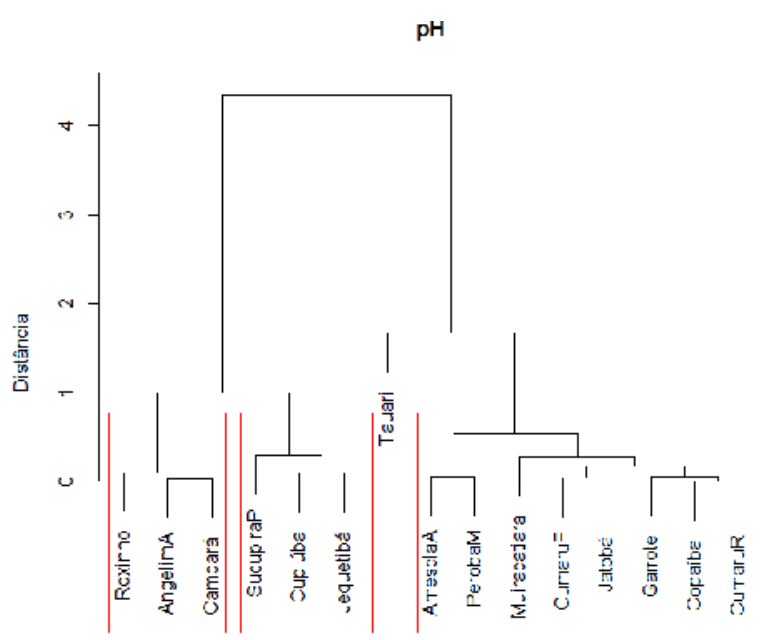

Fapeicies

FIGURA 1. Dendrograma das variáveis extrativos e pH.

A Figura 1 é composta pelo diagrama de extrativos e $\mathrm{pH}$, o mesmo foi dividido em quatros grupos previamente estabelecidos, agrupando-se as espécies com características semelhantes, quanto maior o número de grupos, maior será a semelhança dentro do mesmo, no entanto quanto menos grupos, automaticamente menor será o nível de similaridade.

No dendrograma da Figura 2 as características de semelhança foram baseadas no teor de extrativos que cada espécie apresentou, o primeiro agrupamento (extrema esquerda) é composto por cinco espécies (tauari, cumaru rosa, cambará, peroba miúda e sucupira preta), o segundo agrupamento é formado por apenas uma espécie (copaíba) isso significa dizer que a mesma não possui características de teor extrativo semelhante a nenhuma das outras 14 espécies. $O$ terceiro agrupamento é composto por cinco espécies (cumaru ferro, jatobá, garrote, cupiúba e roxinho), e o último grupo é formado por quatro espécies (amescla aroeira, muiracatiara, angelin amargoso e jequitibá).

Para o diagrama do $\mathrm{pH}$ o primeiro e o segundo agrupamento (extrema esquerda) são compostos por três espécies (roxinho, angelim amargoso e cambará) e (sucupira preta, cupiúba e jequitibá) respectivamente. O terceiro agrupamento é formado por apenas uma espécie (tauari), isso significa que ele não encontrou similaridade com nenhuma das outras espécies, pois o mesmo possui um $\mathrm{Ph}$ de 5,63. O quarto agrupamento é constituído por oito espécies (amescla aroeira, peroba miúda, muiracatiara, cumaru ferro, jatobá, garrote, copaíba e cumaru rosa), esse grupo se destaca por possuir maior quantidade de espécies, visto que a semelhança dentro do grupo ocorreu devido à proximidade dos valores de $\mathrm{pH}(4,4$ a 4,83$)$.

Ao analisar o diagrama (Figura 2) das características físicas, tanto para massa específica básica de partículas, quanto o teor de umidade, o mesmo também foi classificado em quatro grupos, organizado conforme as propriedades físicas. 

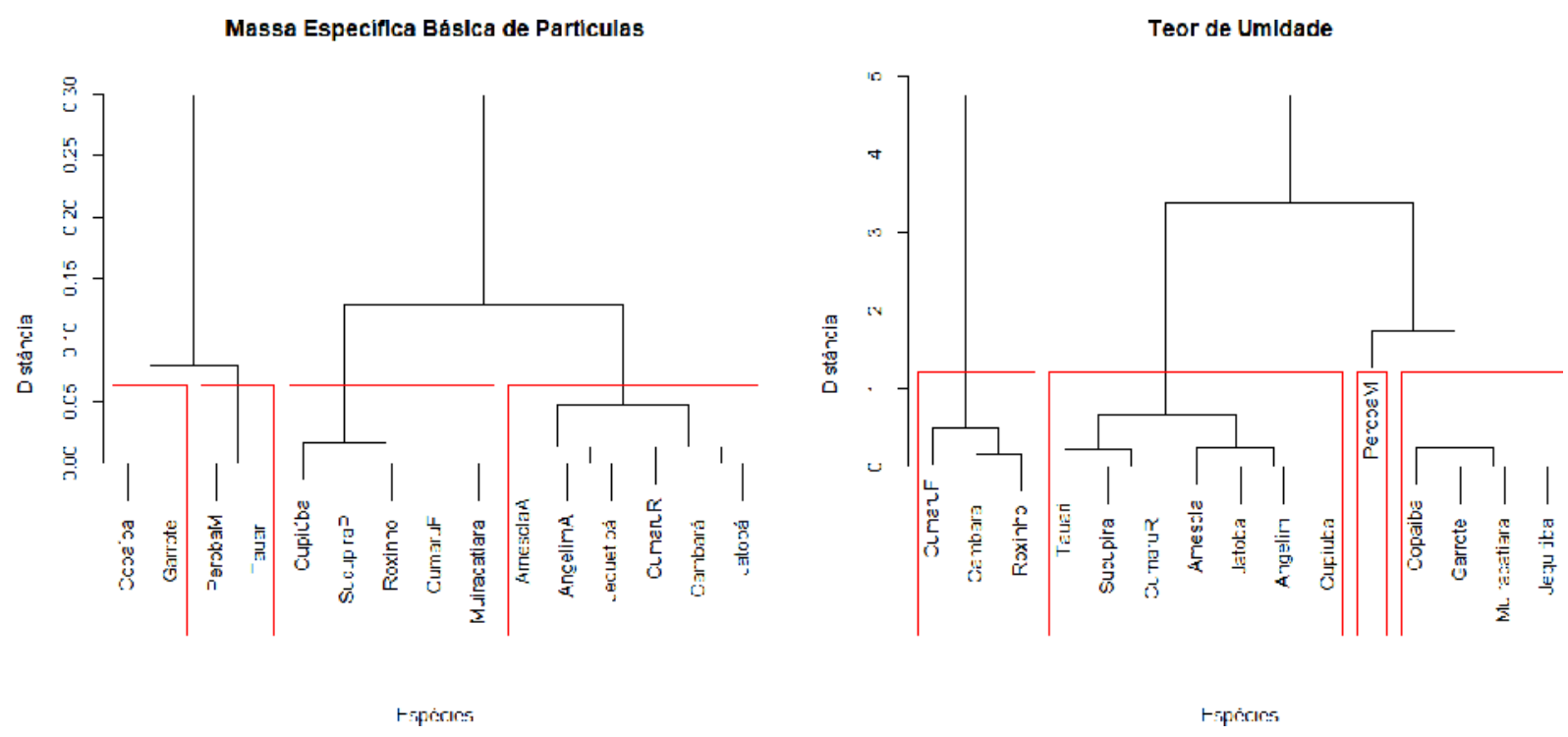

FIGURA 2. Dendrograma das variáveis: massa especifica básica de partículas e teor de umidade.

No dendrograma de massa específica básica das partículas o primeiro e segundo agrupamento (extrema esquerda) é composto por duas espécies (copaíba e garrote) e (peroba miúda e tauari), os níveis possuem uma homogeneidade, visto que a massa para os dois é de $0,19 \mathrm{~g} / \mathrm{cm}^{3}$, assim como também, o grupo das espécies de peroba miúda e tauari ambos com massa especifica básica de 0,15 $\mathrm{g} / \mathrm{cm}^{3}$. O terceiro e quarto agrupamento são constituídos por (cupiubá, sucupira preta, roxinho, cumaru ferro e muiracatiara) e (amescla aroeira, angelim amargoso, jequitibá, cumaru rosa, cambará e jatobá) respectivamente.

Para o teor de umidade foi gerado um diagrama com os respectivos grupos, primeiro (cumaru ferro, cambará e roxinho), segundo (tauari, sucupira preta, cumaru rosa, amescla aroeira, jatobá, angelim amargoso e cupiúba), terceiro (peroba miúda) e quarto agrupamento (copaíba, garrote, muiracatiara e jequitibá). Ressalta-se nesse trabalho, que apesar da ocorrência de espécies da mesma família, em alguns agrupamentos elas ficaram em níveis distintos, mostrando a individualidade de cada gênero estudado.

\section{CONCLUSÕES}

Os ensaios adotados foram adequados para a caracterização física e química das espécies analisadas e a análise de agrupamento possibilitou classificar as madeiras, evidenciando as similaridades ou semelhanças entre elas.

As características estudadas, aliadas a outras propriedades tecnológicas, servirão de subsídio para complementar, aprimorar ou predizer usos futuros para essas espécies, aumentando assim, o leque de madeiras disponíveis como matériaprima para suprir as necessidades humanas.

\section{REFERÊNCIAS}

ABTCP : Associação técnica Brasileira de Celulose e Papel. Normas Técnicas. ABTCP. São Paulo, 1974. 
BRAZ, R. L; OLIVEIRA, J. T. S.; ROSADO, A. M.; VIDAURRE, G. B.; PAES, J. B.; FILHO, M. T.; LOIOLA, P. L. Caracterização anatômica, física e química da madeira de clones de Eucalyptus cultivados em áreas sujeitas à ação de ventos. Ciência da Madeira, Pelotas, RS, v.5, n.2, p. 127-137, 2014. Disponível em: https://periodicos.ufpel.edu.br/ojs2/index.php/cienciadamadeira/article/viewFile/4790/ cienciadamadeirav5n2a7

BRANCALION, P.H.S.; VIANI, R.A.G.; STRASSBURG, B.B.N.; RODRIGUES, R.R. Finding the money for tropical forest restoration, Unasylva, Roma, v. 63, n. 239, p. 41-50, 2012.

CASTRO A. F.N.M.; CASTRO, R.V.O.; CARNEIRO, A.C.O.; LIMA, J.E.; SANTOS R.C.; PEREIRA. B.L.C. Análise multivariada para seleção de clones de eucalipto destinados à produção de carvão vegetal. Pesquisa Agropecuária Brasileira, v. 48, n.6, p. 627-635, 2013. DOI: http://dx.doi.org/10.1590/S0100204X2013000600008.

CHAGAS, S.F.; EVANGELISTA, W. V.; SILVA, J. C.; CARVALHO, A. M. M. L. Propriedades da madeira de teca de diferentes idades e oriundas de desbaste. Ciência da Madeira, Pelotas, RS, v.5 n. 2, p. 138-150, 2014.

Disponível

em:

https://periodicos.ufpel.edu.br/ojs2/index.php/cienciadamadeira/article/viewFile/4806/ cienciadamadeirav5n2a8. DOI: 10.12953/2177-6830.v05n02a08

CIPEM - Centro das Indústrias produtoras e Exportadoras de Madeiras de Mato Grosso. Dados do Setor: Setor de Base Florestal registra crescimento no comércio de produtos. 2015.20 Disponível em: <http://www.cipem.org.br/conteudo.php?sid=44\&cid=1637>. Acesso em: 26 de março de 2018.

FERREIRA, K, A. C; MENDOZA, Z. M. S. H.; RIBEIRO, E. S.; BATISTA, B. M. F.; SILVA, J. C. Análise dos compostos acidentais na madeira de Tectona grandis $\mathrm{L}$. F. Biodiversidade, Cuiabá (MT), v.14, n. 1, p. 105 a 116, 2015.

Disponível

em: http://periodicoscientificos.ufmt.br/ojs/index.php/biodiversidade/article/viewFile/2255/ 1624

FOEKEL, C.E.B. Métodos para determinação de densidade básica de cavacos para coníferas e folhosas. IPEF, Piracicaba, v.2, n. 3, p. 65- 74, 1971.

INPE - Instituto nacional de pesquisas espaciais. Monitoramento da Floresta Amazônica Brasileira por Satélite. Disponível em: <http://www.obt.inpe.br/OBT/assuntos/programas/amazonia/prodes> Acesso em 27 de março de 2018.

MACEDO, M.N.; DEFRIES, R.S.; MORTON, D.C.; STICKLER, C.M.; GALFORD, G.L.; SHIMABUKURO. Decoupling of desforestation and soy production in the southern Amazon during the late 2000s. Proceedings of the National Academy of Sciences of the United States of America, Washington, v. 109, n. 4, p. 1341-1346, 2012. 
MEDEIROS P. N; OLIVEIRA, L; PAES J. B. Relações entre as características da madeira e do carvão vegetal de duas Espécies da Caatinga. Floresta e Ambiente, v. 21, n. 4, p. 484-493, 2014. DOI: http://dx.doi.org/10.1590/2179-8087.051313.

MENDOZA, Z. M. S. H. Tecnologia Química de Produtos Florestais (apostila material didático), editora UFMT, 182p. 2016.

RIBEIRO, E. S.; SOUSA, R. A. T. M.; PAULA, M. H.; MESQUITA, R. R. S.; MOREIRA, E. L.; FAZION, H. Espécies florestais comercializadas pelo estado de Mato Grosso. Revista Biodiversidade, Cuiabá (MT), v. 15, n.2, p. 02-20, 2016.

Disponível

em: http://periodicoscientificos.ufmt.br/ojs/index.php/biodiversidade/issue/view/312/show Toc

SECRETARIA DE FAZENDA DO ESTADO DE MATO GROSSO- SEFAZ. Legislação tributária, ato normativo da SEFAZ. Portaria 12/2015. Institui Lista de Preços Mínimos, para os produtos oriundos da Indústria Florestal e Extrativa Vegetal. Disponível em: <http://app1.sefaz.mt.gov.br/0325677500623408/7C7B6A9347C50F5503256914006 5EBBF/A62B17461A8253E984257DD50041AF75> Acesso em: 27 de março 2018. 